\title{
Indoor wave propagation measurements and modeling for evaluation of coverage enhancement using a repeater system
}

\author{
Jungsuek Oh*, Michael Thiel, Wonbin Hong and Kamal Sarabandi \\ University of Michigan, Ann Arbor, USA \\ jungsuek@umich.edu
}

\section{Introduction}

It is important to know the path loss and field coverage in an indoor environment to be able to set up reliable communication links or a wireless network. The path loss can be measured on site but path loss estimations utilizing numerical methods are faster, cheaper and less time consuming but can still be accurate. For indoor wave propagation, ray-tracing is the usual choice: It allows fast evaluation of large building structures for accurate estimations of the path loss [1] [2]. Furthermore ray-tracing analysis can give insights to the wave propagation phenomena in buildings one can not achieve through measurement.

In the mean time, repeater applications have been proposed in order to enhance the performance of indoor wireless communication [3].

In this paper, we use ray-tracing to analyze indoor wave propagation at $2.4 \mathrm{GHz}$, especially along indoor hallways. Various hallway junctions are analyzed, compared with the actual measurement and the propagation phenomena like multiple corner diffraction along hallways are pointed out. Based on the results, the feasibility of a repeater system to enhance wave propagation along indoor hallways is discussed.

\section{Hallways simulation and measurement results}

The model used for the simulation of hallway propagation is Shoot-and-Bounce Ray-tracing [1]. Fig. 1(a) shows a sample hallway layout (T-shaped). In addition to that, L-shaped and X-shaped hallway junctions are considered as common hallways junctions, as shown in Fig. 2. The path loss along a path from the hallway junction into a hallway with no line of sight $(\mathrm{LoS})$ to the Tx is evaluated. Since transmission through the walls is neglected the received field along the path is solely due to the diffracted field at the corner points. Because of this the results is further compared with single-edge diffraction around one corner to demonstrate how the hallway affects the diffraction around the corner. The path loss was computed along the path R2 of Fig. 2 with a distance of $11 \mathrm{~m}$ between the Tx and the hallway junction at $2.4 \mathrm{GHz}$. The dielectric constant of the wall is 4.8 , representing concrete.

Fig. 1(b) shows the path loss for each case. The received in non-LoS hallways is about $30 \mathrm{~dB}$ higher than in the single diffraction case. This is due to multiple wall reflections in the hallway which leads to multiple diffracted rays to the receiver. Fig. 3 visualized that, while Fig. 3(a) shows the path of single diffraction, Fig. 3(b) shows on of many other ray generated by the multiple reflections in $\mathrm{T}$ - 
corner hallway. In other words, even though the edge diffraction due to the shortest path in hallway is very weak, the edge diffraction of a multiple reflected ray may be strong leading to a much higher received field. In general, the hallway guides the field of the Tx or the corner diffracted field along its path resulting in a higher average field coverage than free space.

In order to verify that the ray-tracing simulations can model a real hallway, measurements were carried out in an actual T-shaped hallway. A real hallway includes cinderblock walls and metal doors. The Tx is put as shown in Fig. 2(a). The received field was recorded along the path R2. Fig. 4 shows the simulated path loss of a T-junction with homogeneous concrete walls, compared with the measurement. The simulation predicts the average path loss well. This result shows that ray-tracing simulations can predict propagation along hallways very well. The differences are mainly due to the unknown dielectric properties of the walls and the hallway features like doors not included in simulation that affect the fast fading.

\section{Repeater system in the hallway}

We introduce the indoor repeater to improve the field coverage by putting it at the center of hallway. We vary the gain of the repeater between $10 \mathrm{~dB}$ and $30 \mathrm{~dB}$ to see the effects on the field coverage. As a simplification of wave propagation using repeater is explained by the Friis formula because the repeater is in a LOS to both Tx and Rx. After the path loss due to the path R1 and R2 is formulated, the gain of repeater is added to it. The equation below shows this progress.

$$
\begin{aligned}
P_{r} & =P_{t} \frac{G_{t} G_{A n t} \lambda^{2}}{\left(4 \pi R_{1}\right)^{2}} G_{A m p} \frac{G_{A n t} G_{r} \lambda^{2}}{\left(4 \pi R_{1}\right)^{2}} \\
& =\left(P_{t} G_{t} G_{r}\right) \frac{\lambda^{2}}{\left(4 \pi R_{1}\right)^{2}} \frac{\lambda^{2}}{\left(4 \pi R_{2}\right)^{2}}\left(G_{A n}^{2} G_{A m p}\right)
\end{aligned}
$$

where $\boldsymbol{G}_{\boldsymbol{A n t}}: \mathrm{Tx} / \mathrm{Rx}$ antenna gain at repeater, $\boldsymbol{G}_{\boldsymbol{A m p}}:$ Amplifier gain at repeater $\boldsymbol{G}^{2}{ }_{A n \boldsymbol{G}} \boldsymbol{G}_{\boldsymbol{A m p}}$ : the total gain of repeater

It is assumed that the repeater simply consists of two Tx and $\mathrm{Rx}$ antennas and one amplifier in the repeater. It should be noted that when wave propagation goes through the repeater, the attenuation due to $\lambda^{2} /(4 \pi)^{2}$ as well as the gain due to

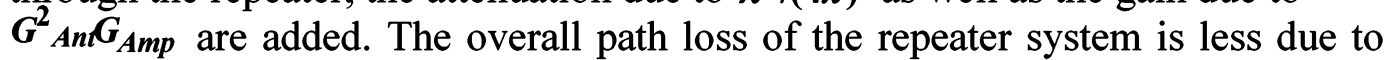
the guiding effects in the hallway. This will be addressed in the talk.

Fig. 5(a) shows the hallway repeater configuration with $\mathrm{R} 1$ as the distance from $\mathrm{Tx}$ to repeater and $\mathrm{R} 2$ as the distance from the repeater to $\mathrm{Rx}$. To calculate the overall path loss of wave propagation including indoor repeater, the gain of repeater is added to the path loss by $\mathrm{R} 1$ and $\mathrm{R} 2$, as shown in equation (2). The gain of repeater varies $10 \mathrm{~dB}$ and $30 \mathrm{~dB}$ and the received power is plotted along the path $\mathrm{R} 2$ with $\mathrm{R} 1=11 \mathrm{~m}$ as shown in Fig. 5(a).

Fig. 5(b) shows the path loss of the repeater system compared with the corner diffraction in the hallway. As can be seen, for a total repeater gain of $20 \mathrm{~dB}$ or higher, the repeater system has the advantage over hallway corner diffraction. 


\section{Conclusion}

In this paper, we demonstrated that hallway propagation is driven by multiple wall reflections leading to high received field in both LoS case and non-LoS case at hallway junctions due to multiple corner diffraction. The result was verified by T-shaped hallway measurements. Based on the simulation results, it was shown that a repeater system with $30 \mathrm{~dB}$ total gain can enhance propagation around hallway corners remarkably.

\section{References:}

[1] S. Y. Seidel, T. S. Rappaport: Site-Specific Propagation Prediction for Wireless In-Building Personal Communication System Design, IEEE TAP, vol. 43, no. 4, pp. 879-891, Nov. 1994

[2] W.D. Burnside, K W. Buergener: High Frequency Scattering by a Thin Lossless Dielectic Slab, IEEE TAP, vol. 31, no. 1, pp. 104-110, Jan 1983 [3] P J Stevens, "Repeaters in PCN and Mobile Cellular Networks," Radio Receivers and Associated Systems, 1995.

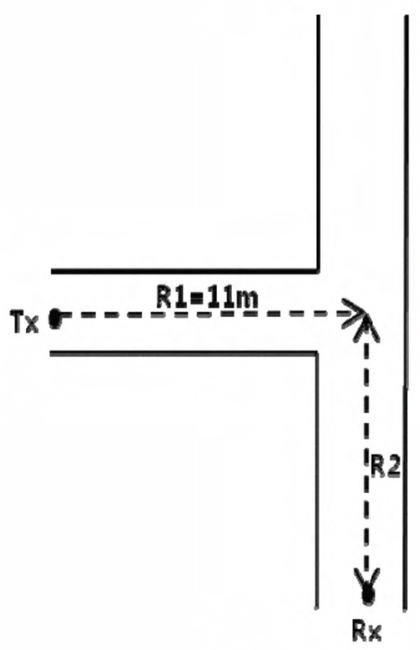

(a)

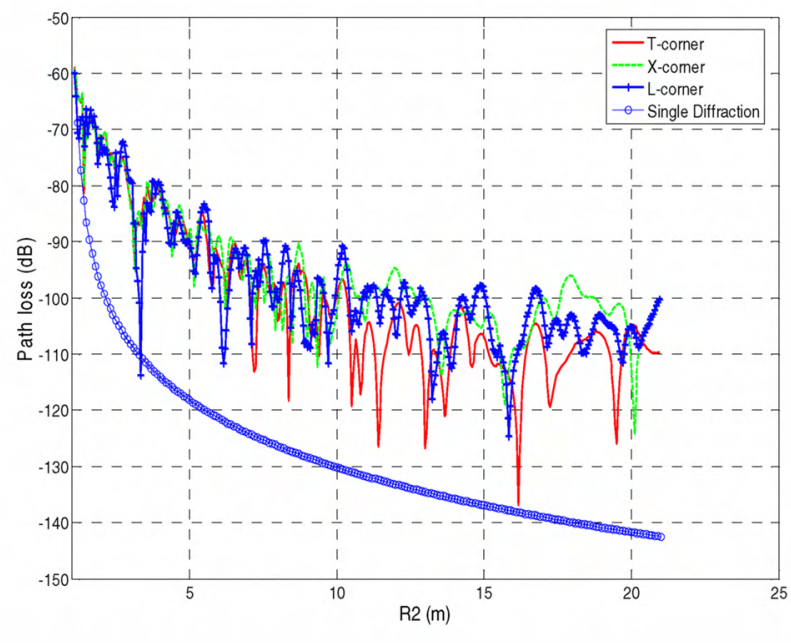

(b)

Fig. 1. (a) Layout for hallways simulation (b) Path loss by various hallways
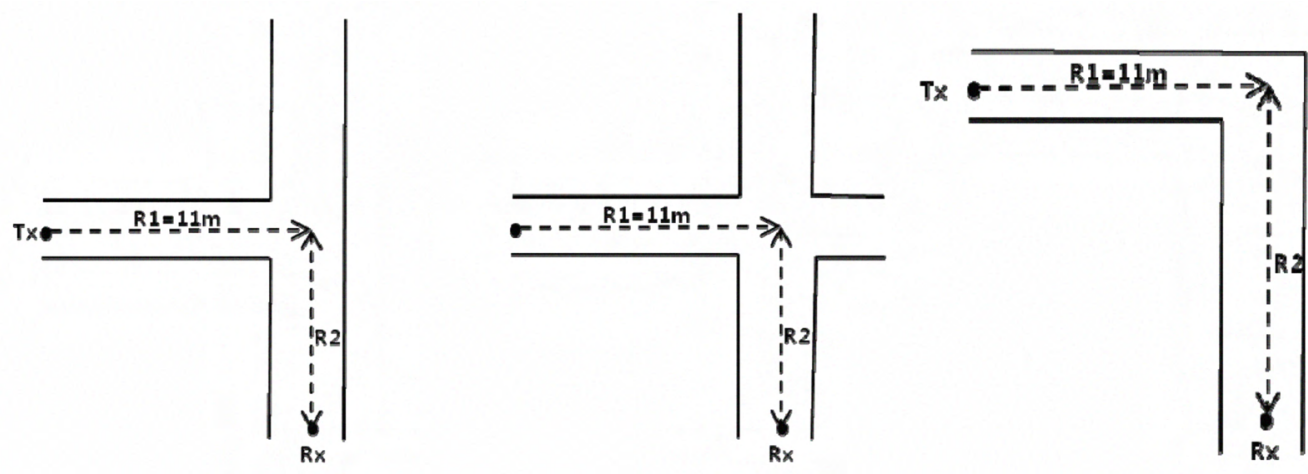

Fig. 2. Hallways with (a) T-type (b) X-type (c) L-type 


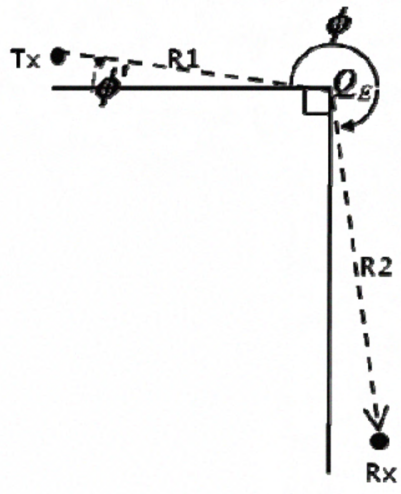

(a)

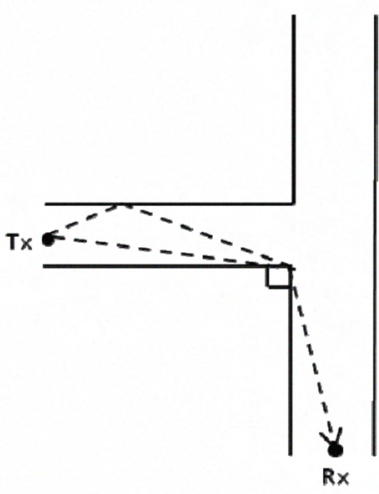

(b)

Fig. 3. Wave Propagation of (a) Single diffraction (b) diffraction in the T-type hallway

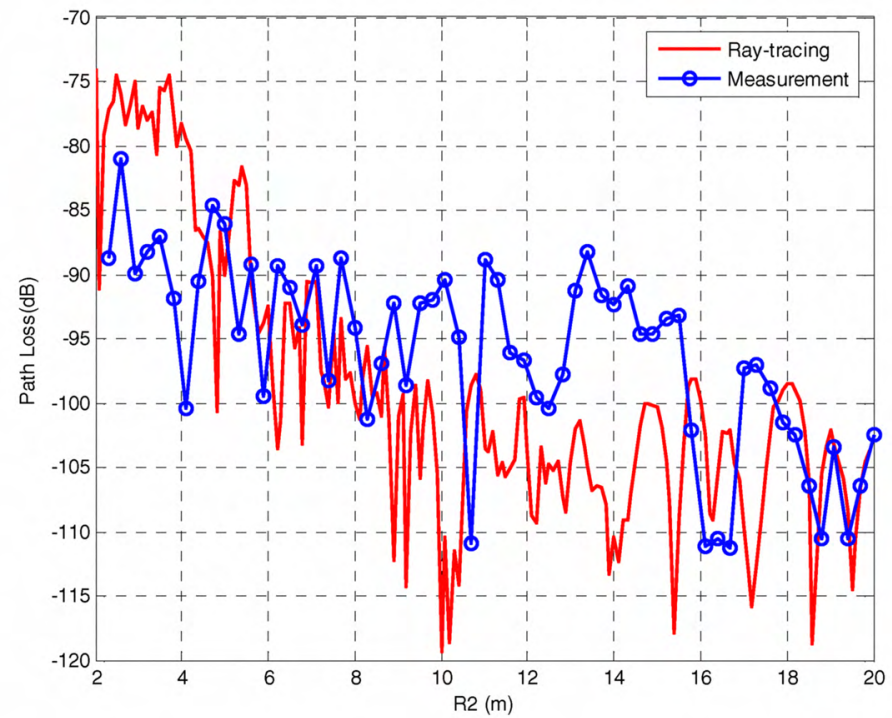

Fig. 4. Ray-tracing Vs. Measurement

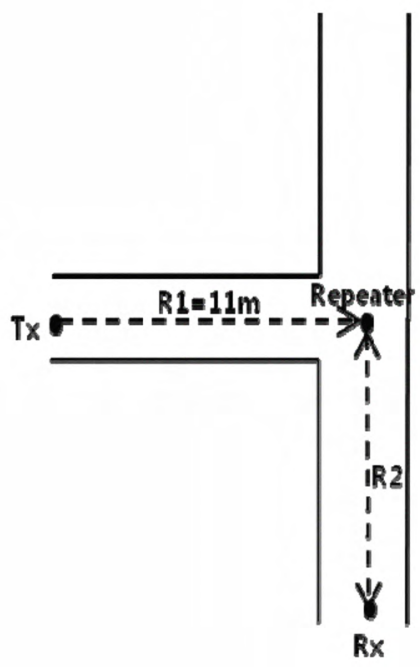

(a)

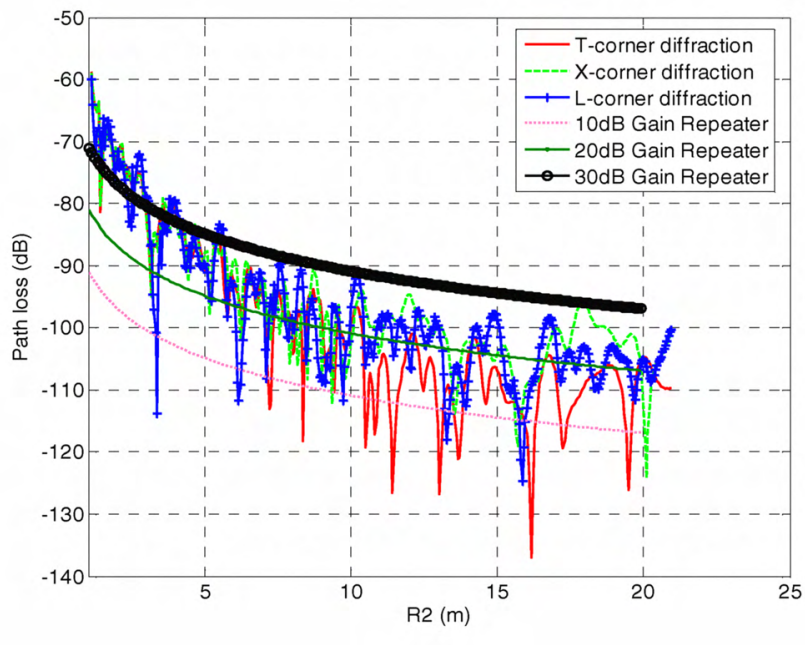

(b)

Fig. 5. (a) Layout for evaluating repeater (b) Path loss by 10, 20 and $30 \mathrm{~dB}$ Repeater Gain 